\title{
Monoenergetic Neutrons for Stellar Applications
}

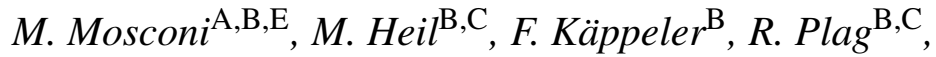 \\ A. Mengoni ${ }^{\mathrm{D}}$, and R. Nolte $\mathrm{A}$ \\ A Physikalisch-Technische Bundesanstalt, Bundesallee 100, 38116 Braunschweig, Germany \\ ${ }^{B}$ Forschungszentrum Karlsruhe, Hermann-von-Helmholtz-Platz 1, \\ 76344 Eggenstein-Leopoldshafen, Germany \\ C Gesellschaft für Schwerionenforschung, Planckstrasse 1, 64291 Darmstadt, Germany \\ D International Atomic Energy Agency, Wagramer Strasse 5, 1400 Vienna, Austria \\ E Corresponding author. Email: marita.mosconi@ptb.de
}

Received 2008 November 15, accepted 2009 February 26

\begin{abstract}
With modern techniques, neutron-capture cross sections can be determined with uncertainties of a few percent. However, Maxwellian averaged cross sections calculated from such data require a correction (because low-lying excited states are thermally populated in the hot stellar photon bath) which has to be determined by theoretical calculations. These calculations can be improved with information from indirect measurements, in particular by the inelastic scattering cross section. For low-lying levels, the inelastically scattered neutrons are difficult to separate from the dominant elastic channel. This problem is best solved by means of pulsed, monoenergetic neutron beams. For this reason, a pulsed beam of $30 \mathrm{keV}$ neutrons with an energy spread of 7 to $9 \mathrm{keV}$ FWHM and a width from 10 to $15 \mathrm{~ns}$ has been produced at Forschungszentrum Karlsruhe using the ${ }^{7} \mathrm{Li}(p, n)^{7} \mathrm{Be}$ reaction directly at the reaction threshold. With this neutron beam the inelastic scattering cross section of the first excited level at $9.75 \mathrm{keV}$ in ${ }^{187} \mathrm{Os}$ was determined with a relative uncertainty of $6 \%$. The use of monoenergetic neutron beams has been further pursued at the Physikalisch-Technische Bundesanstalt in Braunschweig, including the ${ }^{3} \mathrm{H}(p, n)^{3} \mathrm{He}$ reaction for producing neutrons with an energy of $64 \mathrm{keV}$.
\end{abstract}

Keywords: nuclear reactions — nucleosynthesis — scattering

\section{Introduction}

The largest proportion of the nuclei heavier than iron is synthesized via chains of neutron captures. Neutron captures with a very slow rate allow the decay of the generated short-lived nuclei: the so-called $s$ process involves only the nuclei in or close to the valley of stability. Very rapid captures can generate nuclei far from stability that will decay as soon as the neutron irradiation ceases: the $r$ process involves unstable nuclei and its ashes are stable or long-lived nuclei (often also generated by the $s$ process) including the natural actinides. The $s$ process and $r$ process have already been distinguished in the seminal paper on nuclear astrophysics (Burbidge et al. 1957) on the basis of nuclear physics considerations. Meanwhile, the respective astrophysical sites have also been identified: thermally pulsing low mass asymptotic giant branch (AGB) stars for the $s$ process (Busso, Gallino \& Wasserburg 1999) and supernovae (Kratz, Farouqi \& Pfeiffer 2007) or neutron star mergers (Argast et al. 2004) for the $r$ process. Apart from the different neutron densities, the stellar scenarios for the two processes also differ with respect to the prevailing temperatures and the relevant nuclear input. The $r$ process is considered to happen at thermal energies around $k T=100 \mathrm{keV}$ and to reach equilibrium between $(\gamma, n)$ and $(n, \gamma)$ reactions. This means that the Saha equation can be applied (Iliadis 2007) and that the $(\gamma, n)$ and $(n, \gamma)$ cross sections can be neglected except for the freezeout phase (Rauscher 2005). The $s$ process is predicted to occur at lower temperatures (thermal energies from 8 to $25 \mathrm{keV}$ ) and is determined by neutron capture and $\beta$-decay. Accordingly, accurate neutron capture cross section data are required for the $s$-process models.

Such Maxwellian Averaged Cross Sections (MACS) with uncertainties of a few percent can be derived from neutron capture cross sections measured by modern techniques (Ratynski \& Käppeler 1988; Wisshak et al. 1993; Massimi et al. 2008; Tagliente et al. 2008). This is reflected by the recommended experimental $(n, \gamma)$ cross sections compiled in the KADoNiS database, which are given with an average uncertainty of $\pm 6 \%$ (Dillmann 2008).

Under stellar conditions, however, the laboratory cross sections have to be corrected for the effect of low-lying excited states, which can be significantly populated by the hot stellar photon bath. This correction is the so-called Stellar Enhancement Factor (SEF), a multiplicative factor for the laboratory MACS, which has to be determined via theoretical calculations by means of the Hauser-Feshbach statistical model that can usually be applied for heavy nuclei. The reliability of such calculations depends on the quality of the employed parameters. For nuclei in 
the ground state, it has been verified that cross section calculations with global parameters can deviate from experimental data up to a factor of two on average (Bao et al. 2000). Similar uncertainties can be expected for the cross sections of excited nuclear states. Wherever possible, the Hauser-Feshbach parameters should, therefore, be derived from indirect experimental data, e.g. from total cross sections, nuclear level densities, gamma-ray strength functions, and averaged transmission parameters. In this way the reliability of the calculations can be improved to reach 20 to $30 \%$ corresponding to SEF uncertainties of a few percent (Mengoni et al. 2008).

In this context, inelastic scattering cross sections can be used to fine-tune the scattering transmission functions for all scattering channels. Since the scattering channels usually dominate over the capture channel, this information can help to improve the SEF calculations considerably. In particular, the superelastic scattering from an excited state to a lower state can be modeled by means of the inelastic scattering cross section for that excited level (see the example ${ }^{187} \mathrm{Os}^{*}\left(n, n^{\prime}\right)^{187} \mathrm{Os}^{g s}$ as given below $)$.

\section{Measurements of Inelastic Cross Sections}

Inelastic scattering cross sections can be measured either by detecting the scattered neutrons or the $\gamma$-rays from the de-excitation of the reaction nucleus. The latter technique is, in principle, suited for time of flight (TOF) experiments at white neutron source facilities to obtain the cross section over a wide range of neutron energies, but the inelastic cross section has to be extracted from the gamma yields, and these can be impeded for low-lying excited states by electron conversion (Krane 1987) or self absorption.

The detection of scattered neutrons requires a technique to separate the elastic and inelastic components by their different energies. Therefore, a narrow neutron energy distribution is important, which can be achieved by neutron filters or by producing quasi-monoenergetic neutrons via nuclear reactions. This technique has the advantage that it can be safely applied to inelastic scattering from low-lying excited states.

\section{The ${ }^{187}$ Os $\left(n, n^{\prime}\right){ }^{187}$ Os* Cross Section}

The determination of the initial $s$-process abundance of ${ }^{187}$ Os is of particular interest because this nucleus is the daughter of the long-lived radioactive isotope ${ }^{187} \mathrm{Re}$. The $\beta$-decay of ${ }^{187} \mathrm{Re}$ was proposed as a chronometer for the onset of nucleosynthesis in Clayton (1964). Since the first application of this dating technique, which led to inconsistent results (Woosley \& Fowler 1979), large efforts have been spent on solving the issues of the temperature dependence of the ${ }^{187} \mathrm{Re}$ half-life (Bosch et al. 1996) and of the stellar ${ }^{187}$ Os cross section. With respect to the second point, there is the additional complication that the first excited state of ${ }^{187} \mathrm{Os}$ at $9.75 \mathrm{keV}$ is strongly populated at $s$-process temperatures, resulting in a large $\mathrm{SEF}$ correction.

A number of measurements have been reported for determining the $(n, \gamma)$ (Browne \& Berman 1981; Winters
\& Macklin 1982; Bokhovko et al. 1992; Mosconi 2007; Segawa et al. 2007) as well as the inelastic scattering cross section (Macklin et al. 1983; Hershberger et al. 1983; Litvinsky, Murzin \& Shkarupa 1993; Litvinsky et al. 1995; Mosconi et al. 2007b, 2008; Mosconi 2007). Meanwhile, the $(n, \gamma)$ cross section is well-known over the entire range of astrophysical interest, allowing one to calculate the MACS up to $100 \mathrm{keV}$ exclusively from experimental data. The inelastic scattering cross section for the first excited level was measured at $34 \mathrm{keV}$ using a white spectrum combined with a neutron filter (Macklin et al. 1983), ${ }^{3} \mathrm{He}$ counters and filters at 24 and $45 \mathrm{keV}$ (Litvinsky et al. 1993, 1995), and monoenergetic neutron beams at $30 \mathrm{keV}$ (Mosconi 2007; Mosconi et al. 2007b, 2008) and $60 \mathrm{keV}$ (Hershberger et al. 1983). For low-lying excited levels, the separation of elastically and inelastically scattered neutrons becomes difficult because an overlap of both components can hardly be avoided. The TOF technique with a pulsed, monoenergetic neutron beam provides the possibility to identify both components via their different energy distributions.

By fitting the scattering yield with the distributions for the elastically and inelastically scattered neutrons, the measurement of Mosconi (Mosconi 2007; Mosconi et al. $2007 b, 2008$ ) reports an uncertainty of $\pm 6 \%$. The good agreement between four out of five measurements indicates that the problem of the inelastic cross section for the 9.75-keV state in ${ }^{187}$ Os is now satisfactorily settled.

The neutron capture and inelastic scattering cross sections of ${ }^{187}$ Os were used to determine the SEF as well as for estimating the related uncertainty, providing the final stellar MACS with a total uncertainty of 5\% (Mengoni et al. 2008).

\section{Further Data Needs and Experimental Opportunities}

Apart from ${ }^{187}$ Os there are no detailed $\left(n, n^{\prime}\right)$ studies for other nuclei with low-lying excited states. Nevertheless, the growing accuracy of neutron capture measurements and the success of the stellar $s$-process models also requires more accurate information on the SEF data. Excited states with an energy separation lower than $100 \mathrm{keV}$ are not unusual, in particular in the mass range beyond $A \approx 150$, causing sizable SEF corrections especially at higher temperatures.

Also in the field of nuclear technology, the design of the new generation of nuclear power plants requires the update of the nuclear databases (Aliberti et al. 2006). Integral experiments (Salvatores, Aliberti \& Palmiotti 2008) indicate that more precise inelastic scattering cross sections for ${ }^{56} \mathrm{Fe}$ and ${ }^{238} \mathrm{U}$ are important for an improved prediction of the reactor performance. In the frame of the European EFNUDAT (European Facilities for NUclear DATa measurements) project, the feasibility of $\left(n, n^{\prime}\right)$ cross section measurements on ${ }^{56} \mathrm{Fe}$ for the first excited state $(845 \mathrm{keV}$ above the ground state) is presently being investigated at PTB. This measurement will be performed with monoenergetic neutron spectra in the energy range from 2 to 


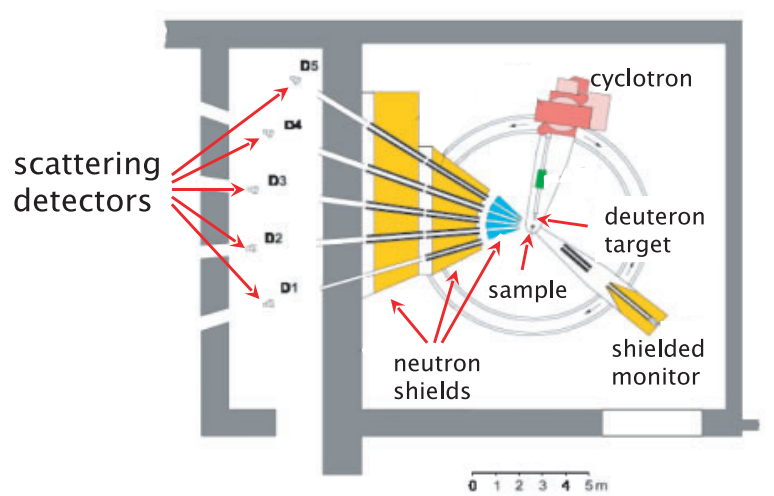

Figure 1 The PTB TOF-spectrometer for neutron scattering experiments. The cyclotron and the shielded monitor are mounted on a circular rail to change the angle with respect to the five detectors (D1 to D5).

$10 \mathrm{MeV}$ using the PTB spectrometer (Schmidt et al. (2005) and see Figure 1).

Neutrons are generated by ${ }^{15} \mathrm{~N}(p, n){ }^{15} \mathrm{O}$ and ${ }^{2} \mathrm{H}(d, n)^{2} \mathrm{He}$ reactions using the proton and deuteron beams from the PTB cyclotron, which is mounted on a circular rail to allow one to measure the scattering cross section in the angular range from $25^{\circ}$ to $160^{\circ}$. Five neutron detectors are used simultaneously and a massive shield avoids the detection of neutrons emitted directly from the deuterium target.

The first excited level in ${ }^{238} \mathrm{U}$ at $45 \mathrm{keV}$ requires a comparably low neutron energy. Monoenergetic beams in this energy regime have not yet been implemented at PTB, but their feasibility is presently being investigated at the 3.75-MV Van-de-Graaff accelerator. Although cross-section data for the first excited state alone are not sufficient for direct technological applications, a precise determination of the elastic and inelastic cross sections at low energy can constrain the extraction of the inelastic cross section at higher energies from $\left(n, n^{\prime} \gamma\right)$ data and for evaluation purposes.

\section{Production of Monoenergetic Neutron Beams in the $\mathrm{keV}$ Range}

Electrostatic accelerators have unique capabilities in terms of energy resolution both in the continuous and the pulsed mode. A resolution of 1 to 2 per thousand for $2-\mathrm{MeV}$ protons is easily achieved at machines equipped with ns pulsing as shown previously at Forschungszentrum Karlsruhe (FZK) as well as at the PTB by means of a 3.75-MV Van-de-Graaff accelerator, and even better values are possible by using sharp energy selection and continuous beams. Although cutting-edge accelerators as radiofrequency quadrupoles can produce much higher currents at the same proton energies, the energy distribution is broader (Praena et al. 2008). This feature makes them less suitable for the production of monoenergetic neutron beams. In fact, the energy spread of the emitted neutrons depends strongly on the monochromaticity and stability of the primary beam. At FZK an energy resolution slightly

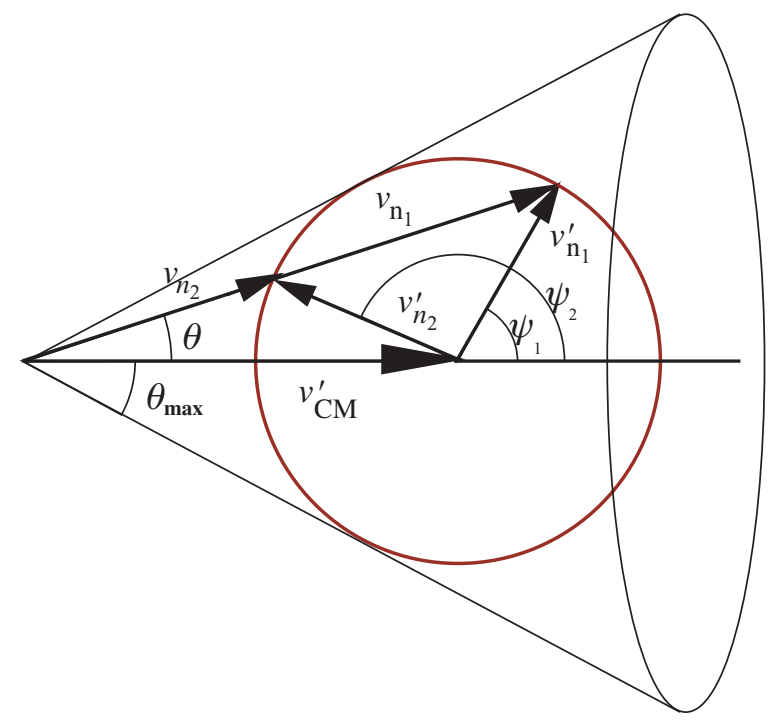

Figure 2 In semi-classical approximation the region of double-valued energy is explained by the composition of velocity vectors. The double-valued energy $\left(E_{\mathrm{n}_{1}}=m_{\mathrm{n}} v_{\mathrm{n}_{1}}^{2} / 2, E_{\mathrm{n}_{2}}=m_{\mathrm{n}} v_{\mathrm{n}_{2}}^{2} / 2\right)$ in the laboratory system occurs when the center-of-mass velocity $v_{\mathrm{cm}}^{\prime}$ exceeds the velocity of the emitted neutron $\left\|\vec{v}_{\mathrm{n}_{1}}^{\prime}\right\|=\left\|\vec{v}_{\mathrm{n}_{2}}^{\prime}\right\|=v_{\mathrm{n}}^{\prime}$. In the center-of-mass system the neutron can take all the directions with an energy $E_{\mathrm{n}}=m_{\mathrm{n}} v_{\mathrm{n}}^{2} / 2$ fixed by the proton energy. The two directions of emission $\psi_{1}$ and $\psi_{2}$ are both transformed in $\theta$, while the correlated velocity $\vec{v}_{\mathrm{n}_{1}}, \vec{v}_{\mathrm{n}_{2}}$ have different magnitude.

better than 2 per thousand and proton currents of typically 3 to $7 \mu \mathrm{A}$ at $1-\mathrm{MHz}$ repetition rate have been used (Mosconi 2002) to generate monoenergetic beams via the ${ }^{7} \mathrm{Li}(p, n){ }^{7} \mathrm{Be}$ reaction.

Directly at the reaction threshold of $E_{\mathrm{p}}=1.8811 \mathrm{MeV}$ the kinematics of ${ }^{7} \mathrm{Li}(p, n)^{7} \mathrm{Be}$ reaction (Monahan 1960) results in the emission of monoenergetic neutrons of $30 \mathrm{keV}$ strictly in forward direction. This energy corresponds to a neutron with zero kinetic energy in the center-of-mass system. With increasing proton energies, neutrons can be emitted in all directions in the centerof-mass system, but in the laboratory system only up to a maximum angle $\theta_{\max }$ that is a function of the proton energy (see Figure 2). The energy of the neutrons can also differ from the threshold value by being both larger and smaller. Two neutron energies (region of double-valued energy) are generated by a singular proton energy at each permitted angle. Always one neutron has an energy larger than $30 \mathrm{keV}$ and the other a smaller, this is often referred as the two energy groups. The region of double-valued energy lasts up to a second threshold called backward threshold. Above this energy neutrons can be emitted in all directions also in the laboratory removing the possibility of two different neutron energies at the same angle. This kinematic behavior is typical of all similar endothermic reactions apart for the values of the quantities involved. The considerations made for the ${ }^{7} \mathrm{Li}(p, n)^{7} \mathrm{Be}$ reaction are valid also for the ${ }^{3} \mathrm{H}(p, n)^{3} \mathrm{He}$ reaction, for instance.

In practice, a sharp proton energy distribution just above threshold generates a neutron beam into a forward cone with a Gaussian energy profile centered at $30 \mathrm{keV}$. 


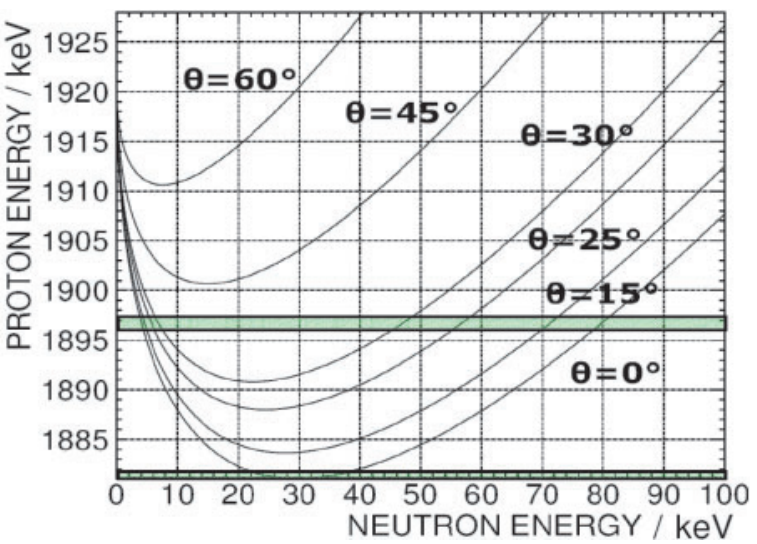

Figure 3 Kinematics of the ${ }^{7} \operatorname{Li}(p, n)^{7} \mathrm{Be}$ reaction, illustrating that quasi monoenergetic neutron beams can be produced by a proton beam with a narrow energy distribution (shadowed areas). At threshold, the neutron energy distribution is only determined by the maximum proton energy whereas the proton energy spread and the angle of emission become important at higher energies.

With a very thin lithium target the interaction energies of the proton beam inside the target can be sufficiently reduced that monoenergetic neutron beams could, in principle, be obtained at restricted emission angles also at higher proton energies (see Figure 3).

At $E_{\mathrm{p}}=1.92 \mathrm{MeV}$ (backward threshold) neutrons can be emitted at angles larger than $90^{\circ}$. Above this energy there is only a single neutron energy at a given angle. The field selection at fixed angles is then completely monochromatic (apart from the contribution from neutron scattering in the target itself) up to the breakup threshold at $3.7 \mathrm{MeV}$. At FZK, neutron energy distributions with a Full Width Half Maximum (FWHM) smaller than $10 \mathrm{keV}$ could be obtained in the region of double-valued energy from $53 \mathrm{keV}$ to roughly $100 \mathrm{keV}$ (Mosconi et al. 2007a). Yet, it is possible to use the kinematic collimation up to the backward threshold at $120-\mathrm{keV}$ neutron energy.

The two energy distributions characteristic of the double-valued energy range are separated by a remaining neutron background. Nevertheless, it is possible to use such beams for measuring inelastic scattering cross sections as demonstrated by Hershberger et al. (1983). This possibility would also be suited for a measurement of the $\left(n, n^{\prime}\right)$ cross section for the $45-\mathrm{keV}$ level in ${ }^{238} \mathrm{U}$. Additionally, the ${ }^{3} \mathrm{H}(p, n)^{3} \mathrm{He}$ reaction can provide suitable energies, e.g. a neutron beam of $63 \mathrm{keV}$ produced at the reaction threshold $\left(E_{\mathrm{p}}=1.019 \mathrm{MeV}\right)$, similar to the case of the ${ }^{7} \mathrm{Li}(p, n)^{7} \mathrm{Be}$ reaction.

\section{PTB Setup}

PTB will continue to reproduce monoenergetic neutrons at the 3.75-MV Van-de-Graaff accelerator. Compared to the performance of the previous FZK machine, the beam current is smaller (around $1 \mu \mathrm{A}$ at $1.25 \mathrm{MHz}$ ) due to the compact internal pulsing system. The pulsing and the klystron bunching systems use two apertures where a large part of the beam is lost. The klystron reduces the time spread of the beam to around 2 to $3 \mathrm{~ns}$ from the typical time spread of about $10 \mathrm{~ns}$ behind the first pulsing section. The klystron itself reduces the beam intensity by only $40 \%$ but induces an additional energy spread of the proton beam, which was found to increase the FWHM of the neutron energy distributions by $25 \%$.

The current can easily be doubled by using a pulsing frequency of $2.5 \mathrm{MHz}$. The possibility to increase the beam current using a larger aperture or singular deflection for the pulsing are under discussion. It has been found, however, that a simple reduction of the deflection of the beam by the pulsing system disturbs the symmetry of the time distribution of the pulse. On the other hand, the beam energy appears to be more stable than at FZK and the experimental hall is designed to generate less scattering. Lithium targets of different types can be employed (metallic lithium and $\mathrm{LiF}$ ), but for the ${ }^{3} \mathrm{H}$ target only a tritium loaded titanium backing is available.

\section{First Test and Results}

In the first beam time at PTB the Gaussian shape of the neutron spectra emitted at the threshold of the two $(p, n)$ reactions was tested. The neutron spectrum was measured by means of a Li glass scintillator placed in front of the target at a flight path of $30 \mathrm{~cm}$. The klystron bunching system was in use for all the spectra shown. The targets consisting of a $70 \mu \mathrm{g} \mathrm{cm}^{-2} \mathrm{LiF}$ layer and a ${ }^{3} \mathrm{H}$ loaded Ti layer of $853 \mu \mathrm{g} \mathrm{cm}^{-2}$, both evaporated onto a $0.5-\mathrm{mm}$ silver backing. Neutrons were detected with a NE912 Li glass monitor $3.81 \mathrm{~cm}$ in diameter and $3 \mathrm{~mm}$ in thickness, mounted on a XP2020 photomultiplier tube. The time information was obtained by coupling the anode signal to a CANBERRA constant fraction discriminator. This signal was the start of an ORTEC 567 time to analog converter and the stop was the pickup signal from the accelerator. The beam current was between 0.8 and $1.2 \mu \mathrm{A}$ at $1.25 \mathrm{MHz}$ or twice that much at $2.5 \mathrm{MHz}$.

In Figure 4, a typical energy spectrum for the ${ }^{3} \mathrm{H}(p, n){ }^{3} \mathrm{He}$ reaction is shown. The centroid is not perfectly at $64 \mathrm{keV}$ because it corresponds to a thick target yield obtained with a proton energy $5 \mathrm{keV}$ above the reaction threshold. The small ${ }^{3} \mathrm{H}(p, n)^{3} \mathrm{He}$ cross section does not permit one to measure closer to the threshold. The energy resolution of the beam is acceptable for a measurement of inelastic scattering on ${ }^{238} \mathrm{U}$, but the registered count rate of six neutrons per second allows one to measure only in conditions of very low background.

A corresponding neutron distribution obtained at PTB at the threshold of the ${ }^{7} \mathrm{Li}(p, n)^{7} \mathrm{Be}$ reaction is shown in Figure 5. At that time we were not able to reproduce the result of FZK in terms of count rate. However, the use of an $\mathrm{LiF}$ target, the smaller current, and the use of the klystron explain this result.

\section{Conclusions and Outlook}

Inelastic scattering measurements for the first excited level are nowadays possible even for low-lying states. PTB is committing some effort to developing a dedicated facility 


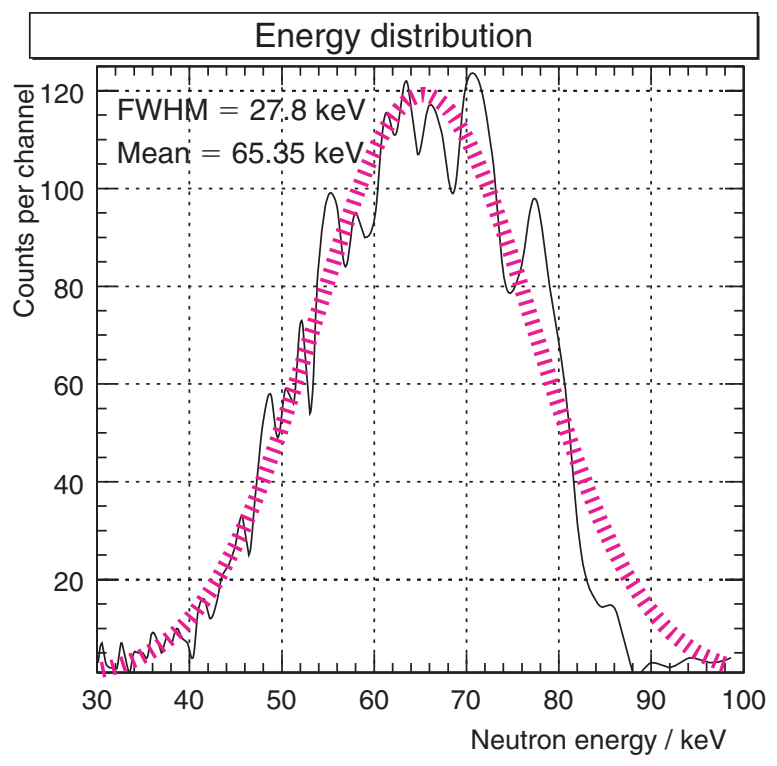

Figure 4 Neutron energy distribution obtained with a proton beam $5 \mathrm{keV}$ above the threshold of the ${ }^{3} \mathrm{H}(p, n)^{3} \mathrm{He}$ reaction.

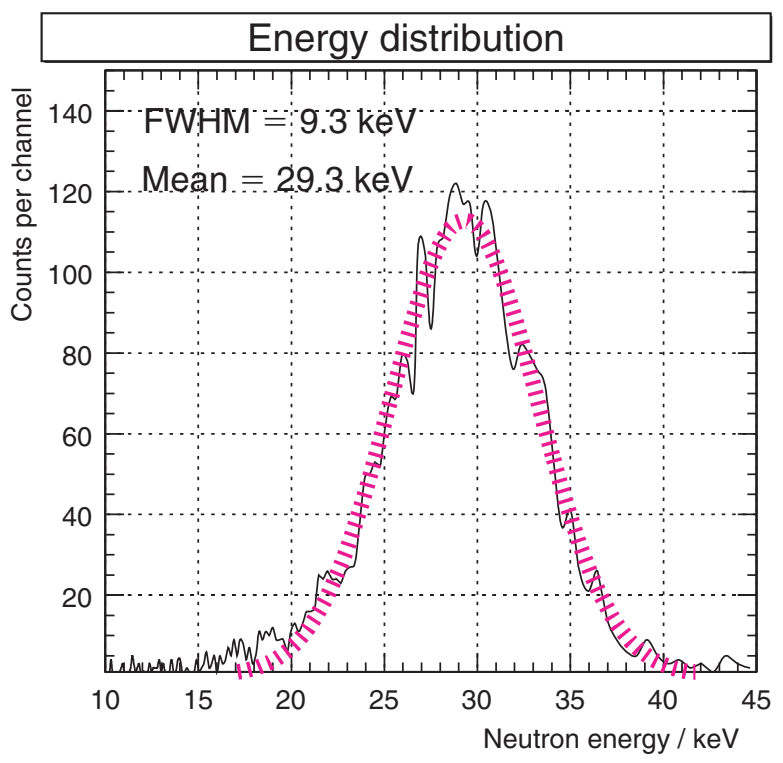

Figure 5 Neutron energy distribution obtained at the threshold of the ${ }^{7} \mathrm{Li}(p, n)^{7} \mathrm{Be}$ reaction.

with neutron beams from 30 to $120 \mathrm{keV}$. Test runs with thin ( $7 \mu \mathrm{g} \mathrm{cm}^{-2}$ corresponding to $1-\mathrm{keV}$ proton energy loss) and thick $\left(50 \mu \mathrm{g} \mathrm{cm}^{-2}\right.$ corresponding to $7-\mathrm{keV}$ proton energy loss) metallic lithium targets are already scheduled. The possibility to increase the proton current is presently being considered, and the background in the experimental area during the measurements is under investigation. Checking whether the low-scattering experimental area of PTB allows one to perform measurements at count rates lower than in previous experiments will be undertaken.

Measurements of the inelastic scattering for excited levels some hundred $\mathrm{keV}$ above the ground state are presently possible from 2 to $14 \mathrm{MeV}$.

\section{Acknowledgments}

This work has been financed by the EFNUDAT project. We are grateful to D. Roller, E. P. Knaetsch and W. Seith of FZK, and to W. Beverung, H. Eggestein, T. Heldt, M. Hoffmann, S. Khurana, S. Löb and M. Thiemig of PTB, for providing continuous support at the two 3.7-MV Vande-Graaff accelerators.

\section{References}

Aliberti, G. et al., 2006, AnNE, 33, 700

Argast, D., Samland, M., Thielemann, F. K. \& Qian Y. Z., 2004, A\&A, 416, 997

Bao, Z. Y., Beer, H., Käppeler, F., Voss, F., Wisshak, K. \& Rauscher, T., 2000, ADNDT, 76, 70

Bokhovko, M., Kononov, V., Poletaev, E., Rabotnov, N. \& Timokhov, V., 1992, in Nuclear Data for Science and Technology, Ed. Qaim, S. (Berlin: Springer), 62

Bosch, F. et al., 1996, PhRvL, 77, 5190

Browne, J. C. \& Berman, B. L., 1981, PhRvC, 23, 1434

Burbidge, E. M., Burbidge, G. M., Fowler, W. A. \& Hoyle, F., 1957, RvMP, 29, 547

Busso, M., Gallino, R. \& Wasserburg, G., 1999, ARA\&A, 37, 239

Clayton, D. D., 1964, ApJ, 139, 637

Dillmann, I., 2008, AIPC, 1016, 143

Hershberger, R., Macklin, R., Balakrishnan, M., Hill, N. \& McEllistrem, M., 1983, PhRvC, 28, 2249

Iliadis, C., 2007, Nuclear Physics of Stars (Wenheim, Germany: Wiley-VCH)

Krane, K. S., 1987, Introductory Nuclear Physics (John Wiley and Sons)

Kratz, K.-L., Farouqi, K. \& Pfeiffer, B., 2007, PrPNP, 59, 147

Litvinsky, L. L., Murzin, A. V. \& Shkarupa, A. M., 1993, PAN, 56, 1161

Litvinsky, L. L., Zhigalov, Y. A., Libman, V. A., Murzin, A. V. \& Shkarupa, A. M., 1995, PAN, 58, 164

Macklin, R., Winters, R., Hill, N. \& Harvey, J., 1983, ApJ, 274, 408

Massimi, C. et al., 2008, in Proc. Int. Conf. Nuclear Data for Science and Technology, Eds. Bersillon, O., Gunsing, F., Bauge, E., Jacqmin, R. \& Leray, S. (EDP Sciences), 1265

Mengoni, A. et al., 2008, in Proc. NICX, Proceedings of Science, in press

Monahan, J., 1960, in Fast Neutron Physics, part I, Eds. Marion, J. B. \& Fowler, J. L. (New York: Interscience Publishers Inc.)

Mosconi, M., 2002, Master's Thesis, Università degli Studi di Torino

Mosconi, M., 2007, PhD Thesis, Karlsruhe Universität

Mosconi, M., Plag, R., Heil, M., Käppeler, F. \& Mengoni, A., 2007, in Proc. 8th Int. Topical Meeting on Nuclear Applications and Utilization of Accelerators (American Nuclear Society), 807

Mosconi, M. et al., 2007b, PrPNP, 59, 165

Mosconi, M. et al., 2008, JPhG, 35, 014015

Praena, J., Mastinu, P. F., Calviani, M. \& Martin, G., 2007, LNL Annual Report 2008, 214

Ratynski, W. \& Käppeler, F., 1988, PhRvC, 37, 595

Rauscher, T., 2005, NuPhA, 758, 655

Salvatores, M., Aliberti, G. \& Palmiotti, G., 2008, in Proc. Int. Conf. Nuclear Data for Science and Technology, Eds. Bersillon, O., Gunsing, F., Bauge, E., Jacqmin, R. \& Leray, S. (EDP Sciences), 883

Schmidt D. et al., 2005, NIMPA, 545, 658

Segawa, M. et al., 2007, PhRvC, 76, 022802

Tagliente, G. et al., 2008, in Proc. Int. Conf. Nuclear Data for Science and Technology, Eds. Bersillon, O., Gunsing, F., Bauge, E., Jacqmin, R. \& Leray, S. (EDP Sciences), 1304

Winters, R. R. \& Macklin, R. L., 1982, PhRvC, 25, 208

Wisshak, K., Guber, K., Voss, F., Käppeler, F. \& Reffo, G., 1993, PhRvC, 48, 1401

Woosley, S. E. \& Fowler, W. A., 1979, ApJ, 233, 411 